\title{
DEGS1-associated aberrant sphingolipid metabolism impairs nervous system function in humans
}

\author{
Gergely Karsai, ${ }^{1,2}$ Florian Kraft, ${ }^{3}$ Natja Haag, ${ }^{3}$ G. Christoph Korenke, ${ }^{4}$ Benjamin Hänisch, ${ }^{3}$ Alaa Othman, ${ }^{1,2}$ \\ Saranya Suriyanarayanan, ${ }^{1,2}$ Regula Steiner, ${ }^{1,2}$ Cordula Knopp, ${ }^{3}$ Michael Mull, ${ }^{5}$ Markus Bergmann, ${ }^{6}$ J. Michael Schröder, ${ }^{7}$ \\ Joachim Weis, ${ }^{7}$ Miriam Elbracht, ${ }^{3}$ Matthias Begemann, ${ }^{3}$ Thorsten Hornemann, ${ }^{1,2}$ and Ingo Kurth ${ }^{3}$ \\ 'Center for Integrative Human Physiology, University of Zürich, Zürich, Switzerland. "2nstitute for Clinical Chemistry, University Hospital, Zürich, Switzerland. ${ }^{3}$ Institute of Human Genetics, Medical Faculty, \\ RWTH Aachen University, Aachen, Germany. ${ }^{4}$ Clinic for Neuropediatrics and Congenital Metabolic Diseases, University Clinic for Paediatrics and Adolescent Medicine, Oldenburg, Germany. ${ }^{5}$ Department \\ of Diagnostic and Interventional Neuroradiology, Medical Faculty, RWTH Aachen University, Aachen, Germany. ${ }^{6}$ Institute for Neuropathology, Hospital Bremen-Mitte, Bremen, Germany. ${ }^{7}$ Institute of \\ Neuropathology, Medical Faculty, RWTH Aachen University, Aachen, Germany.
}

BACKGROUND. Sphingolipids are important components of cellular membranes and functionally associated with fundamental processes such as cell differentiation, neuronal signaling, and myelin sheath formation. Defects in the synthesis or degradation of sphingolipids leads to various neurological pathologies; however, the entire spectrum of sphingolipid metabolism disorders remains elusive.

METHODS. A combined approach of genomics and lipidomics was applied to identify and characterize a human sphingolipid metabolism disorder.

RESULTS. By whole-exome sequencing in a patient with a multisystem neurological disorder of both the central and peripheral nervous systems, we identified a homozygous p.Ala280Val variant in DECS1, which catalyzes the last step in the ceramide synthesis pathway. The blood sphingolipid profile in the patient showed a significant increase in dihydro sphingolipid species that was further recapitulated in patient-derived fibroblasts, in CRISPR/Cas9-derived DECS1-knockout cells, and by pharmacological inhibition of DECS1. The enzymatic activity in patient fibroblasts was reduced by $80 \%$ compared with wild-type cells, which was in line with a reduced expression of mutant DECS1 protein. Moreover, an atypical and potentially neurotoxic sphingosine isomer was identified in patient plasma and in cells expressing mutant DECS1.

CONCLUSION. We report DECS1 dysfunction as the cause of a sphingolipid disorder with hypomyelination and degeneration of both the central and peripheral nervous systems.

TRIAL REGISTRATION. Not applicable.

FUNDING. Seventh Framework Program of the European Commission, Swiss National Foundation, Rare Disease Initiative Zurich.

\section{Introduction}

Sphingolipids (SLs) are fundamental components of eukaryotic cell membranes where they play crucial roles in membrane architecture and signaling (1). They are major components of the myelin sheath and of fundamental importance for neural function (2). Whereas oligodendrocytes support and insulate neurons of the central nervous system, equivalent function is provided by Schwann cells in the peripheral nervous system. Besides a role in myelin sheath formation and maintenance, signaling SLs such

Authorship note: GK, FK, and $\mathrm{NH}$ contributed equally to this work. TH and IK contributed equally to this work.

Conflict of interest: The authors have declared that no conflict of interest exists. License: Copyright 2019, American Society for Clinical Investigation.

Submitted: August 10, 2018; Accepted: December 21, 2018.

Reference information: / Clin Invest. 2019;129(3):1229-1239.

https://doi.org/10.1172/JCl124159. as sphingosine-1-phosphate (S1P) and ceramide-1-phosphate are bioactive lipid hormones that regulate a variety of physiological functions. Ceramides are central components of SL metabolism, as they form the building blocks for complex SLs like sphingomyelin (SM) and glycosylceramides. They also represent the crossroad for the degradation and salvage pathways (3). Ceramide biosynthesis starts at the endoplasmic reticulum (ER) with the conjugation of L-serine and palmitoyl-CoA, the rate-limiting step catalyzed by serine palmitoyltransferase (SPT). The immediate product 3-keto-sphinganine is reduced to sphinganine (SA), which is then $\mathrm{N}$-acylated to dihydroceramide (dhCer) by 1 of 6 ceramide synthase isoforms (CerS1-6) (4). In the final step, dhCer is converted to ceramide by the insertion of a $\Delta 4,5$ trans $(\Delta 4 \mathrm{E})$ double bond into the SA backbone. This final conversion is catalyzed by the $\Delta 4$-dihydroceramide desaturase DEGS1 (5). On the catabolic side, ceramides are deacylated by ceramidases to form sphingosine (SO), which can be either recycled back to ceramides (sal- 

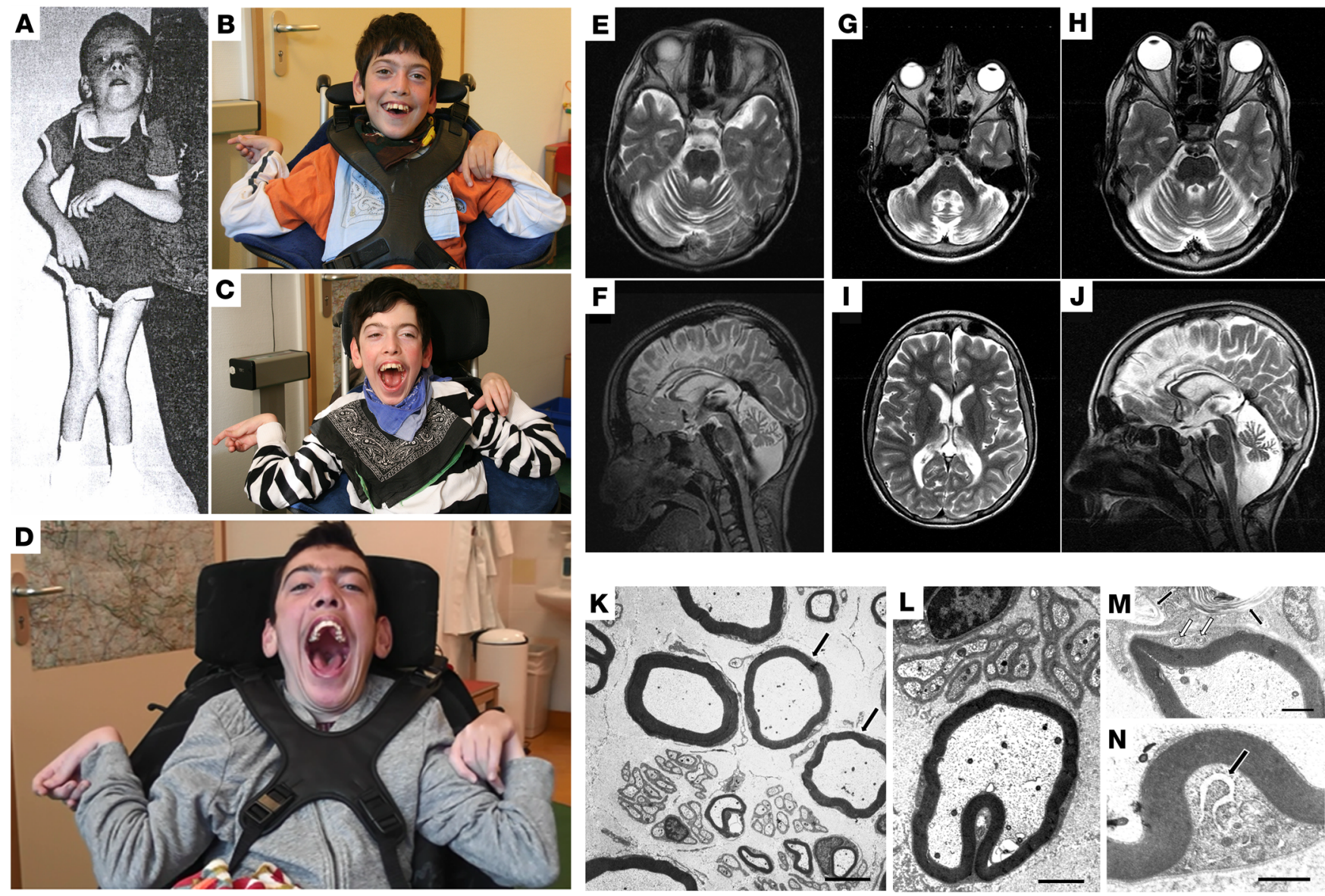

O

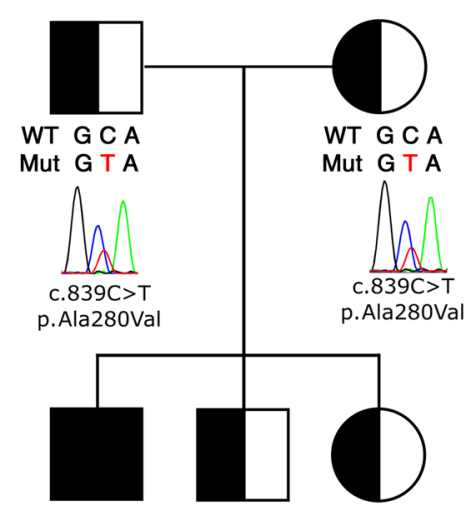

Mut $\mathbf{G} T \mathbf{A}$

Mut GTA

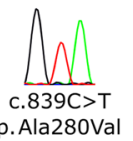

P DEGS1 (NP_003667.1)

Sphingolipid Delta(4)-Desaturase DES1 isoform 1

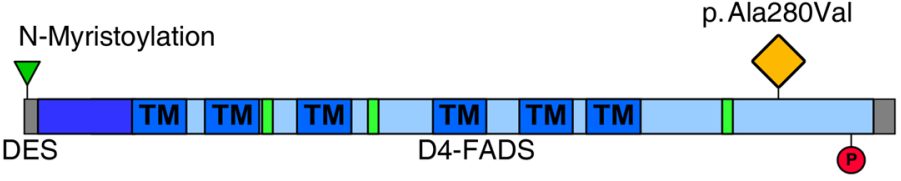

DES: Sphingolipid-Delta4 desaturase (aa 6-42)

D4-FADS: Delta4-Sphingolipid-FADS-like (light blue, aa 26-315)

Histidine boxes (green, aa 89-93/128-132/259-263)

TM: transmembrane domain

P: phosphorylation site (aa 307)

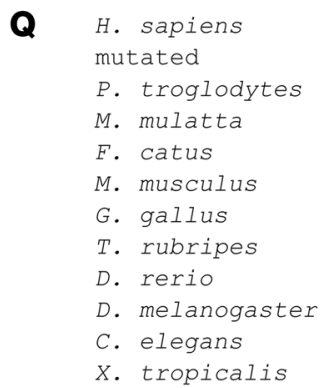

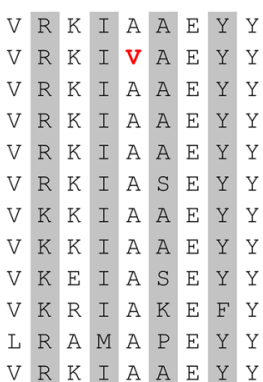


Figure 1. Clinical phenotype and genetics of the DECS1 disorder. Clinical phenotype with progression of spasticity, notably in the arms and hands. Patient at the age of 6 years (A), 13 years (B), 15 years (C), and at last followup at 22 years (D). T2-weighted MRI of the brain, axial (E, and $\mathbf{G}-\mathbf{I}$ ) and sagittal (F and $\mathbf{J})$, at 11 years of age (E and $\mathbf{F}$ ) and 16 years (G-J). Severe and slowly progressive cerebellar atrophy with fiber degeneration of the middle cerebellar peduncles. The patient shows mild cortical atrophy and thin white matter, especially in the posterior brain regions. In summary, MRI findings are in line with a progressive global neurodegenerative process. (K-N) Electron micrographs of the sural nerve biopsy performed at the age of 2 years reveals nerve fibers with disproportionately thin myelin sheaths (K, arrows). Scale bar: $3 \mu \mathrm{m}$. (L) Occasional, moderate myelin folding. Scale bar: $1.8 \mu \mathrm{m}$. (M) Small autophagic vacuoles in the cytoplasm of the Schwann cell of a myelinated nerve fiber (white arrows) Black arrows indicate large autophagic vacuoles containing membranous debris in an adjacent cell, which is covered by a basal lamina and may therefore be either a Schwann cell or a macrophage that has invaded a Schwann cell basal lamina sheath. Scale bar: $0.75 \mu \mathrm{m}$. (N) Widening of the endoplasmic reticulum (arrow) of a Schwann cell. Scale bar: $0.5 \mu \mathrm{m}$. (0) The pedigree of the family shows the segregation of the DEGS1 variant [NM_003676.3:c.839C>T, p.Ala280Val, Chr1(hg19):g.224380047C>T].

Sanger traces of the affected codon are shown in the index patient and his parents. (P) Domain architecture of the human DEGS1 protein. Position of the mutation is indicated in orange. (Q) Species alignment of the amino acid residues in proximity of the DECS1 mutation. Mutation highlighted in red. FADS, fatty acid desaturase domain.

vage pathway) or phosphorylated by SO kinases (SK1/SK2) to form sphingosine-1-phosphate (S1P). S1P is a potent lipid hormone that binds to specific S1P receptors (SP1R1-6), which control a multitude of cellular responses (6). S1P can either be converted back to SO through action of S1P phosphatases (S1PPase), or terminally degraded by the S1P lyase (SGPL1) to hexadecenal and ethanolamine phosphate (7). For complex SL formation, ceramides are transported from the ER to the Golgi and are converted into phosphosphingolipids (e.g., SM) or glycosphingolipids (GalCer, GluCer). Subsequently, they are metabolized to highly complex glycosphingolipids such as gangliosides (8).

The degradation of complex SLs requires dedicated catabolic enzymes, such as glycohydrolases and sphingomyelinases that reside in the plasma membrane, ER, Golgi apparatus, and lysosomes $(9,10)$. Defects in these catabolic enzymes cause sphingolipidoses such as Fabry, Gaucher, Farbers, Niemann-Pick, and Tay-Sachs (11). Defects in the synthesis pathway are also associated with disease. Mutations in SPT (SPTLC1 and SPTLC2) lead to the formation of aberrant and neurotoxic 1-deoxysphingolipids, which result in hereditary sensory and autonomic neuropathy type I (OMIM \#162400, \#613640) (12). Genetic variants in ceramide synthase 1 and 2 (CERS1/2) are associated with progressive myoclonic epilepsy, generalized tonic-clonic seizures, tremor, dysarthria, ataxia, and developmental delay (OMIM \#616230) (13-15). Mutations in the GM3 synthase gene (ST3GAL5) lead to refractory epilepsy, psychomotor delay, blindness, and deafness (OMIM \#609056) $(16,17)$, and GM2/GD2 synthase mutations (B4GALNT1) lead to GM3 accumulation and a complex form of hereditary spastic paraplegia with cognitive impairment and seizures (OMIM \#609195) (18). Recently, mutations in SGPL1 were associated with a broad spectrum of disease phenotypes including recessive steroid-resistant nephrotic syndrome (SRNS), ichthyosis, adrenal insufficiency, immunodeficiency, and brain defects
(OMIM \#617575) (19-21), but also with axonal peripheral neuropathy without renal or adrenal deficiencies (22).

Here, we identify DEGS1 dysfunction as the cause of an SL disorder with leukodystrophy and hypomyelination of the peripheral nervous system.

\section{Results}

Clinical description and genetic analysis. The 22-year-old male patient was the first born of healthy consanguineous Turkish parents and showed a progressive mixed pyramidal and extrapyramidal movement disorder as well as a progressive cerebellar atrophy. At the age of 6 months a motor developmental delay was observed and progressive spasticity became obvious in the subsequent clinical course (Figure 1, A-D, and Supplemental Video; supplemental material available online with this article; https://oi.org/10.1172/ JCI124159DS1). Consecutive brain MRI revealed a general hypomyelination, a thinning of the brainstem and occipital white matter, severely reduced volume of both thalami, progressive cerebellar and supra- and infratentorial atrophy, and a thin corpus callosum, most pronounced in the dorsal part (Figure 1, E-J). In the clinical course, he developed a pathological EEG with epilepsy and grand mal seizures, which were successfully treated by a combination of valproate and carbamazepine. He showed a progressive neurological dysfunction, microcephaly, dystrophy, a progressive scoliosis, neurogenic bladder, and gastroesophageal reflux. Since the age of 18 years, feeding required a percutaneous endoscopic gastrostomy. Progressive spasticity resulted in flexion contractures of the extremities, a positive Babinski sign, and increased muscle tone. At the age of 19 years, intrathecal baclofen pump therapy was initiated. Detailed clinical findings are summarized in Table 1. A muscle and sural nerve biopsy was performed at the age of 2 years. Archived electron micrographs (Figure 1, K-N) from the sural nerve biopsy showed several nerve fibers with disproportionately thin myelin sheaths, moderate myelin folding, widening of the ER of Schwann cells, and several autophagic vacuoles in the cytoplasm of Schwann cells. The muscle biopsy revealed neurogenic muscular atrophy according to the records that could be retrieved; however, no muscle specimens were available for review. Electroneurography at both arms and legs showed significantly slowed nerve conduction velocities, with only a slight reduction of the amplitudes, in line with a predominant demyelinating neuropathy. Metabolic screening for lysosomal storage disorders did not show pathological findings. Genetic workup revealed a normal male karyotype (46, XY) and array-CGH was unsuspicious (data not shown).

Using whole-exome sequencing in the index patient, his 2 unaffected siblings, and both parents revealed a suspicious homozygous missense variant in the index patient in DEGS1 (NM_003676.3) (Figure 1, O-Q and Supplemental Table 1). Both parents and the siblings were heterozygous carriers of this DEGS1 variant (Figure 1O). The variant changes codon 280 from alanine to valine (p.Ala280Val) (Figure 1P), affecting a highly conserved nucleotide $(c .839 \mathrm{C}>\mathrm{T}$ ) and amino acid (Figure 1Q). The altered residue is located in the fatty acid desaturase/SL $\Delta 4$-desaturase domain. The variant was not present in public databases (dbSNP, 1000 Genomes, ESP server, ExAC, and gnomAD) and was predicted to be deleterious by several bioinformatics pathogenicity pre- 


\section{Table 1. Clinical findings in the affected individual}

\begin{tabular}{|c|c|}
\hline Parameters & Findings \\
\hline DEGS1 (NM_003676.3) & c.839C>T; p.Ala280Val \\
\hline Chromosomal position (hg19) & chr1:224380047C>T \\
\hline Origin & Turkey (consanguineous parents) \\
\hline Gender & Male \\
\hline Age at onset & 6 months \\
\hline Age at last followup & $21 \mathrm{yr}$ \\
\hline Clinical diagnosis & $\begin{array}{l}\text { Multisystem disorder with progressive tetraspasticity, } \\
\text { epilepsy, mental retardation, microcephaly }\end{array}$ \\
\hline $\begin{array}{l}\text { Clinical phenotype at last } \\
\text { followup }\end{array}$ & $\begin{array}{l}\text { Intellectual disability, nonambulatory, severe spasticity, } \\
\text { nonverbal, friendly }\end{array}$ \\
\hline Body weight & $\begin{array}{l}\text { Normal at birth, dystrophy since the age of } 3 \mathrm{yr} \text {; at last followup } \\
43 \mathrm{~kg}(-3.7 \mathrm{SD}) ; \text { percutaneous gastrostomy since age } 18 \mathrm{yr}\end{array}$ \\
\hline Body length & $\begin{array}{l}\text { Normal at birth, mild short stature since the age of } 4.5 \mathrm{yr} \text {; } \\
\text { at last followup } 160 \mathrm{~cm}(-2.5 \mathrm{SD})\end{array}$ \\
\hline Head circumference & $\begin{array}{l}\text { Normal in the first year then progressive microcephaly; } \\
\text { at last followup } 53 \mathrm{~cm}(-2.5 \mathrm{SD})\end{array}$ \\
\hline Epilepsy & $\begin{array}{l}\text { Onset } 5 \text { years, grand mal epilepsy; under therapy with valproate } \\
\text { and carbamazepine, no seizures since age } 11 \mathrm{yr}\end{array}$ \\
\hline Brain MRI & $\begin{array}{c}\text { Cerebellar atrophy, mild global atrophy, thin white matter } \\
\text { especially in the posterior brain regions }\end{array}$ \\
\hline Visual evoked potentials & Pathological latency \\
\hline Acoustic evoked potentials & Pathological latency \\
\hline BERA & Pathological interpeak latency/auditory threshold 85 db \\
\hline Electroneurography & $\begin{array}{l}\text { Mild demyelinating neuropathy with decreased mNCV: } \\
\text { medianus mNCV } 30.0 \mathrm{~m} / \mathrm{s} \text { age } 2 \mathrm{yr}, 28.7 \mathrm{~m} / \mathrm{s} \text { age } 12 \mathrm{yr} \text {; } \\
\text { tibialis mNCV } 38.0 \mathrm{~m} / \mathrm{s} \text { age } 2 \mathrm{yr}, 39.2 \mathrm{~m} / \mathrm{s} \text { age } 12 \mathrm{yr}\end{array}$ \\
\hline Nerve biopsy & Peripheral hypomyelination \\
\hline Muscular biopsy & Neurogenic muscular atrophy \\
\hline Scoliosis & Scoliosis surgery at age $18 \mathrm{yr}$ \\
\hline Neurogenic bladder disorder & Present \\
\hline
\end{tabular}

BERA, brainstem evoked response audiometry; mNCV, motoric nerve conduction velocity; yr, years.

diction tools (CADD phred [score 35], SIFT [score 0], Polyphen2 HDIV [score 0.99], and MutationTaster prediction [score D]).

Splicing, subcellular localization, and expression of mutant DEGS1. Besides a codon change from alanine to valine (p.Ala280Val), the software tools NNSplice and GeneSplicer predicted a possible splicing effect for the c.839C > T variant in DEGS1. To address an influence on splicing, we used third-generation longread nanopore sequencing of cDNA from patient fibroblasts and did not observe aberrant transcripts compared to cDNA from control fibroblasts and reference transcripts (Figure 2A).

To investigate whether the p.Ala280Val variant (mut DEGS1) affects subcellular localization, we overexpressed EGFP-tagged wild-type (WT) and mut DEGS1 in HeLa cells. Cells were costained with markers for the ER and mitochondria (Figure 2, B-E). Both WT and mut DEGS1 colocalized with the ER marker protein disulfide isomerase (PDI) (Figure 2, B and C) and showed only little colocalization with the mitochondrial marker Tim23 (Figure 2, D and E). Notably, DEGS1 expression levels were reduced in patient fibroblasts compared with controls, as determined by Western blot (Figure 2, F and G). To further analyze the DEGS1 mutation, DEGS1 and the homologous DEGS2 were knocked out in HAP1 cells by CRISPR/Cas9 technology (DEGS1/-, $\left.D E G S 2^{-/-}\right)$. Similarly to the expression in fibroblasts, overexpression of mut DEGS1-EGFP in HAP1 cells resulted in a lower protein amount compared with WT DEGS1-EGFP (Figure 2, $\mathrm{H}$ and I). FACS measurements of HAP1 WT cells transfected with WT or mut DEGS1 fused to EGFP in addition showed a lower number of GFP-positive cells (Supplemental Figure 1). Moreover, inhibiting protein translation with cycloheximide in HAP1 WT cells overexpressing either WT or mut DEGS1-EGFP indicated a reduced half-life of the mutant. In contrast, treating cells with the proteasome inhibitor MG-132 stabilized both WT and mut DEGS1 protein (Figure 2, H and J). This implies that the mutation p.Ala280Val affects overall DEGS1 protein stability.

$S L$ analysis. We performed an untargeted lipidomics analysis from plasma of the index patient, his parents, and 6 unrelated controls. Total SL plasma levels were comparable between the individuals; however, the patient plasma revealed a striking overrepresentation of dihydrosphingolipid (dhSL) species (dhSM, dhCer, and dhHexCer) compared with parents and controls (Figure $3 \mathrm{~A})$. The relative proportion of dhSLs in patient plasma was approximately $40 \%$, while it was about $10 \%$ in controls and parents. This disproportion was confirmed in patient-derived skin fibroblasts. SL de novo synthesis was measured by culturing the fibroblasts in the presence of stable isotope-labeled d4-serine, which is incorporated in de novo-formed sphingoid bases. Like in plasma, we observed a significantly increased formation of dhSL species in patient fibroblasts compared with control cells (Figure 3B). To confirm that this shift is directly caused by DEGS1 dysfunction, we used the DEGS1 and DEGS2-deficient HAP1 lines. In $\mathrm{DEGS1}^{-/-}$cells, approximately $90 \%$ of the de novo-formed SLs were present in the saturated dihydro form, whereas $D E G S 2^{-1-}$ cells showed no changes in the profile. Interestingly, the relative levels of dhSLs were lower in patient-derived fibroblasts (40\%) compared with $\mathrm{DEGS1}^{- \text {-- }}$ cells (90\%), suggesting that the DEGS1 p.Ala280Val mutant may have some residual activity. We therefore analyzed DEGS1 activity in patient fibroblasts by measuring the conversion of stable isotope-labeled d7-sphinganine (d7SA) to d7-sphingosine ( $\mathrm{SO}+7)$ (Figure $3 \mathrm{C}$ ). In patient fibroblasts, a slow conversion between the 2 forms was observed, indicating that the p.Ala280Val mutant still has some activity. Compared with control fibroblasts, the rate was approximately 5 -fold lower, indicating a residual activity of approximately $20 \%$. This remaining activity was completely suppressed in the presence of the DEGS1 inhibitor fenretinide (4-HPR) (Figure 3C).

DEGS1 deficiency results in the formation of a potentially novel sphingoid base. Surprisingly, when analyzing the sphingoid base profile in hydrolyzed plasma samples we observed a second peak that was isomeric to SO. The peak was detected in the patient plasma but not in plasma of the parents or unrelated controls (Figure 

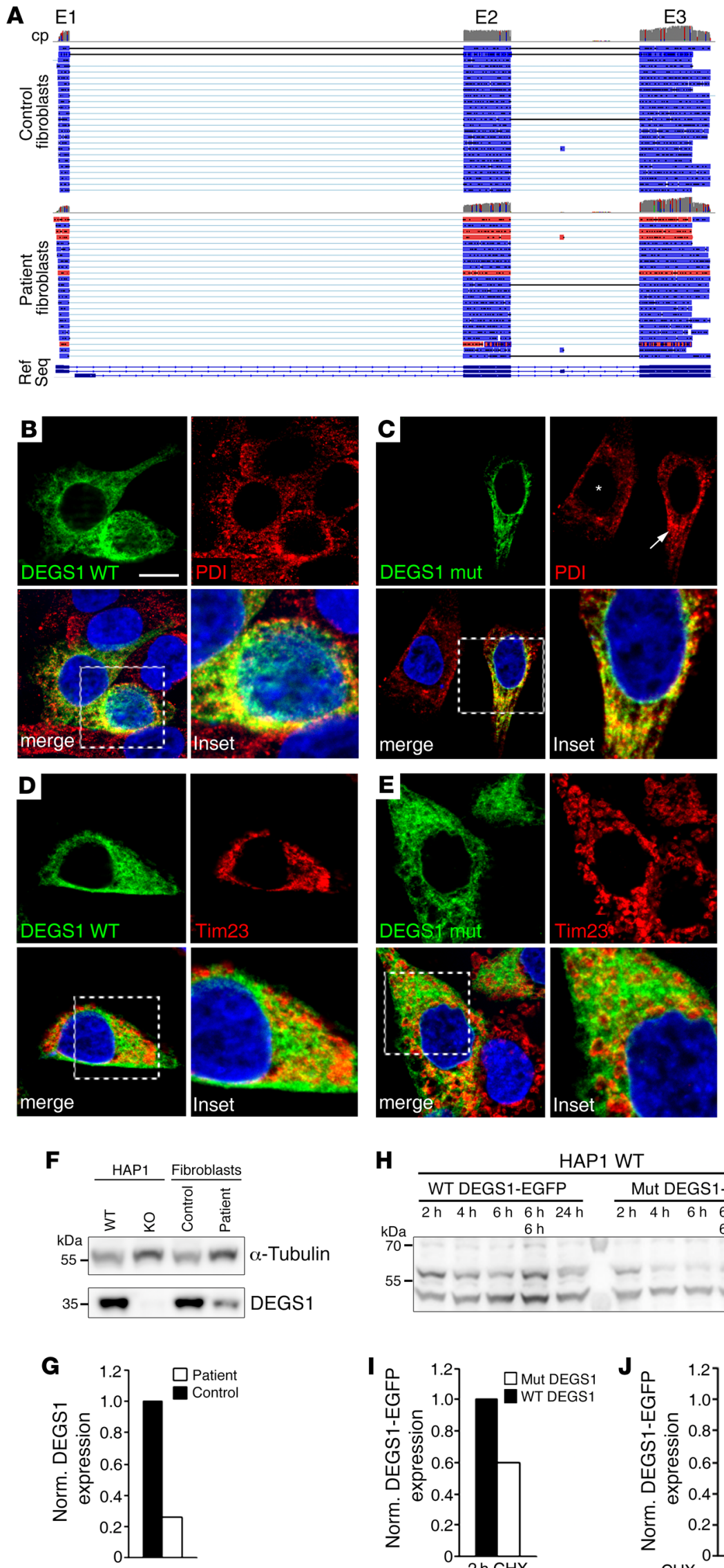

H

HAP1 WT

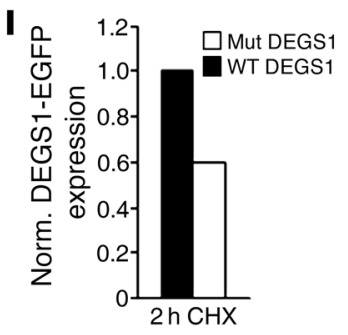

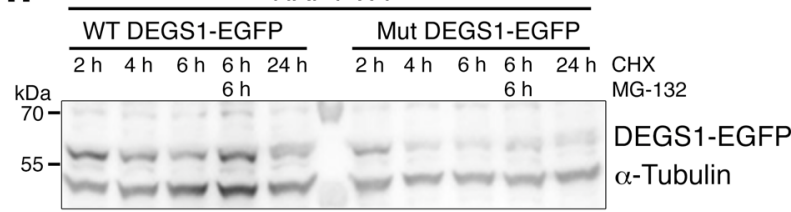

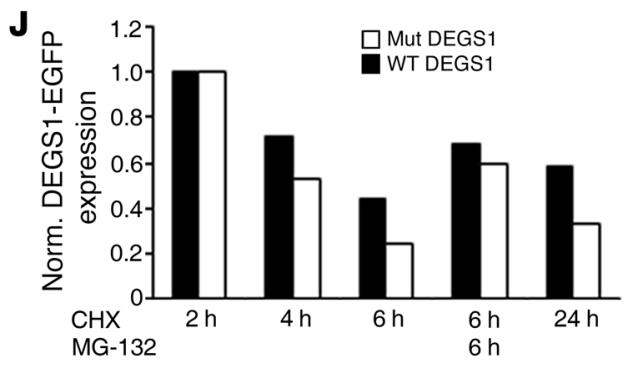



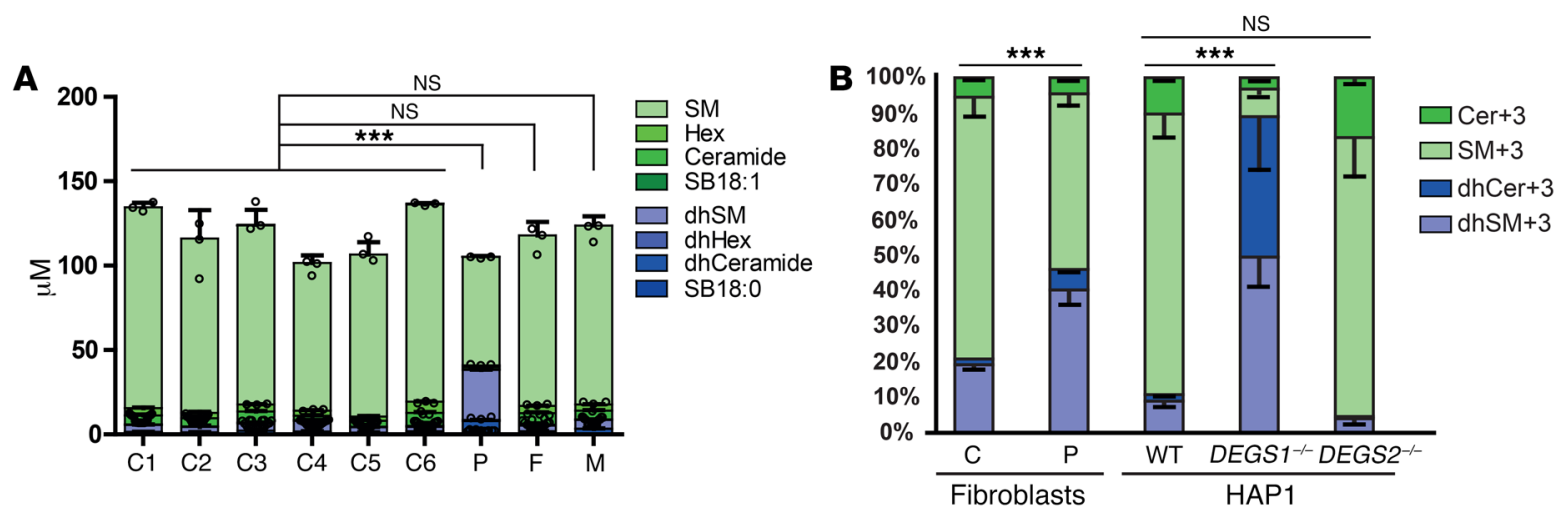

C

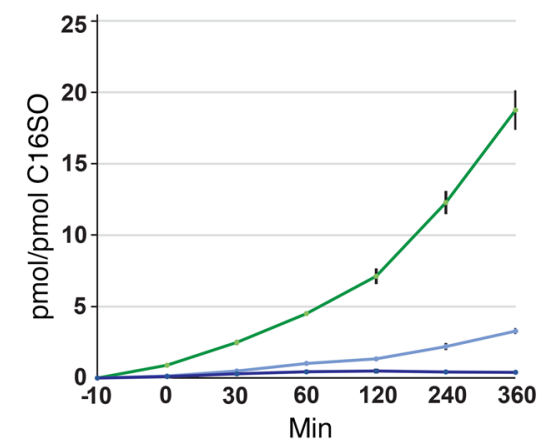

D

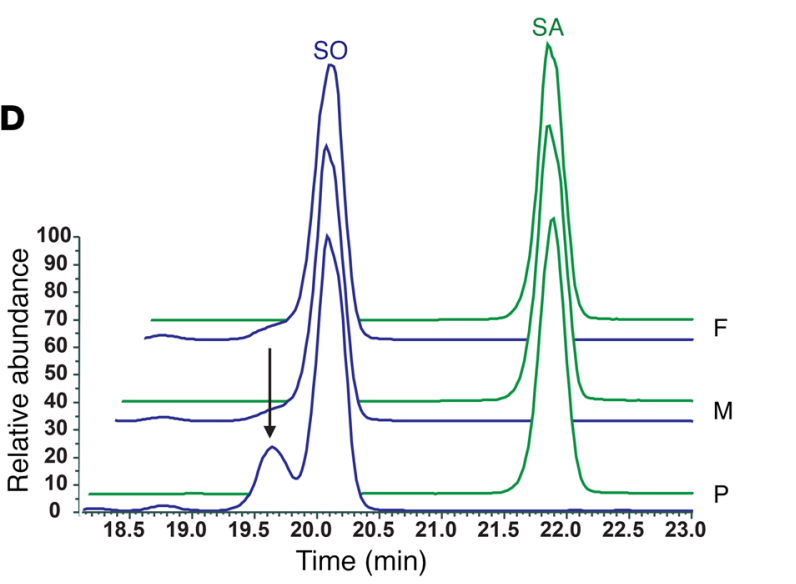

Figure 3. Lipidomics analysis of mutant DEGS1. (A) Lipidomics analysis showed a significant elevation of dhSL species (dhCer, dhSM, and dhHexCer) in patient plasma (P) compared with parents (F, M) or unrelated controls (C1-6). (B) Cultured patient-derived fibroblasts showed an increase in de novosynthesized dhSL (dhCer+3 and dhSM+3) compared with cells from unrelated controls. Increased dhSL levels were also seen in DECS1 ${ }^{-1-}$ HAP1 cells where the dhSL species reached up to $90 \%$ of the total SLs. In contrast, WT cells had less than $15 \%$ dhSL species. Slightly decreased dhSL levels were observed in DECS2 ${ }^{-1-}$ cells. (C) Kinetics of the DEGS1 reaction in control and patient fibroblasts. Cells were supplemented with $2 \mu \mathrm{M}$ d7SA (arrow) and the increase in total SO+7 was followed over time. Values were normalized to internal C16SO levels (ISTD). In patient-derived fibroblasts, DECS1 activity was 5-fold lower compared with controls. This residual activity was fully inhibited in the presence of the DECS1 inhibitor 4-HPR ( $2 \mu \mathrm{M})$. (D) The sphingoid-base profile after hydrolysis revealed an isomeric SO metabolite (arrow) with an approximately 30-second-shorter retention time. The metabolite could be detected in the patient plasma but not in plasma of the parents or unrelated controls. No isomeric peak was seen for SA (green). $n=3$; data presented as the mean \pm SD or -SD. ${ }^{* *} P<0.001$ by 1 -way ANOVA with Tukey's correction for multiple testing.

3D). The metabolite had the same mass as SO, but a 30-secondearlier retention time. To confirm that this peak is a bona fide sphingoid base, we performed an isotope labeling assay, supplementing d4-serine to HAP1 WT and DEGS1 ${ }^{-/}$cells. This resulted in the formation of canonical SO+3 in HAP1 WT cells and in the +3-labeled isomeric peak in $D E G S 1^{-1}$ cells (Figure $4 \mathrm{~A}$ ). The same labeling pattern was observed in control fibroblasts when DEGS1 activity was inhibited with 4-HPR (Figure 4A), confirming that this metabolite is directly associated with reduced DEGS1 activity. Furthermore, the metabolite was not formed when SPT activity was inhibited with myriocin, indicating that the formation is downstream of SPT (Supplemental Figure 2A). To gain further insight into the metabolic origin of this atypical sphingoid base, we supplemented DEGS1-cells with isotope-labeled d7SA or d7SO. In d7SA-supplemented cells the isomeric metabolite was formed quantitatively and isotope labeled $(+7)$, whereas $\mathrm{d} 7 \mathrm{SO}$ was not converted and reappeared in the cells as $\mathrm{SO}+7$ with the expected retention time for the canonical SO. This indicated that the metabolite is indeed a downstream product of SA and not a direct product of SPT (Figure 4B). A struc- tural analysis using chemical derivatization with dimethyl disulfide followed by collision-induced HCD fragmentation (23) revealed a specific fragment $(\mathrm{m} / z$ 110.10156), reflecting an isotope-labeled 4 -carbon tail fragment of SO+7 (Figure 4C). An analogous nonlabeled fragment was also identified in SO-supplemented cells (Supplemental Figure 2B). These results suggest that the double-bond position of the isomeric SO metabolite is in the $\Delta 14$ position and distinct from the $\Delta 4$ position of canonical SO. This result was further confirmed by supplementing DEGS1 $1^{-/}$cells with $\mathrm{d} 4\left(\mathrm{C}_{11}-\mathrm{C}_{12}\right)-$ labeled palmitate. When incorporated into the sphingoid base, the $\mathrm{d} 4\left(\mathrm{C}_{11}-\mathrm{C}_{12}\right)$-labeled palmitate is converted to $\mathrm{SA}+4$ with four deuterium labels at the $\mathrm{C}_{13}-\mathrm{C}_{14}$ position (Supplemental Figure $2 \mathrm{C}$ ). Introducing a $\Delta 4$ double bond will not affect the label, whereas a $\Delta 14$ double bond results in the loss of 1 deuterium. As expected, canonical SO $(\Delta 4)$ was found to be exclusively +4 labeled (Supplemental Figure $2 \mathrm{C}$ ), whereas the isomeric pre-peak contained the +3 label, which further supports a $\Delta 14$ position of the double bond.

Supplementation of an SL-rich diet as a therapeutic intervention. Correcting the altered dhSL/SL ratio in the patient was considered 
A

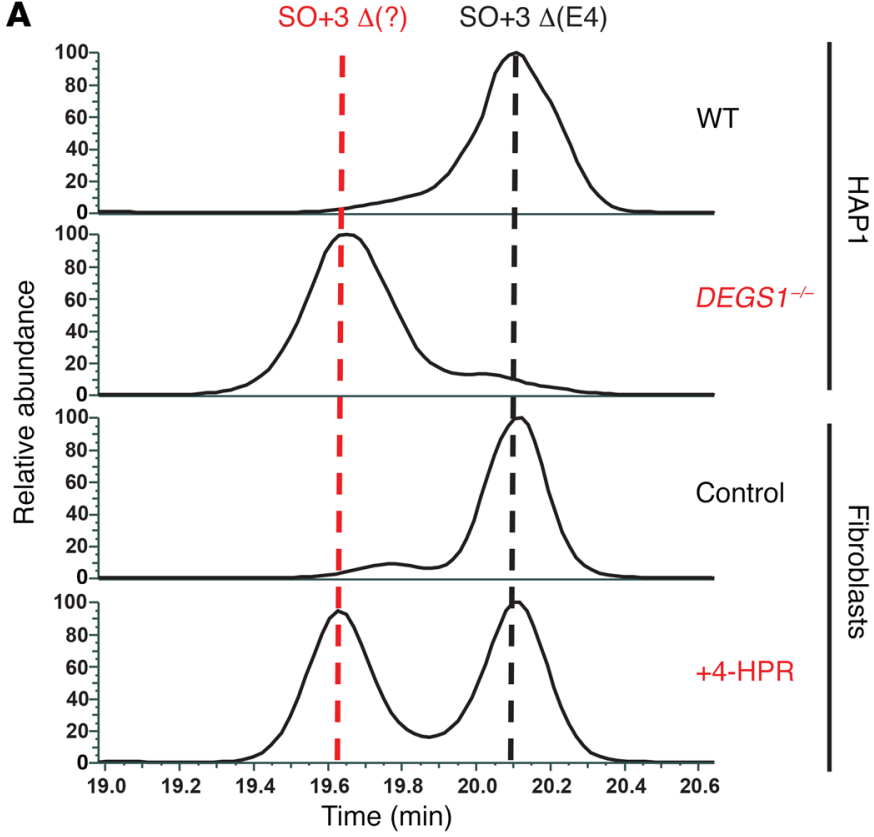

B

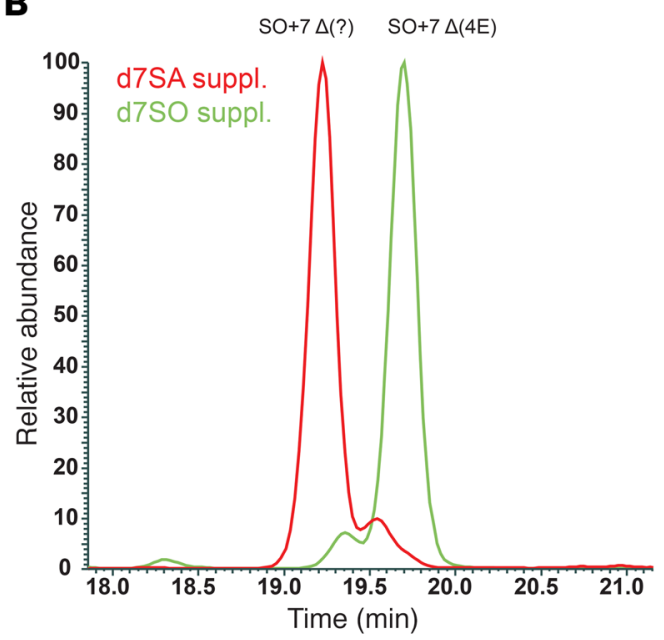

C

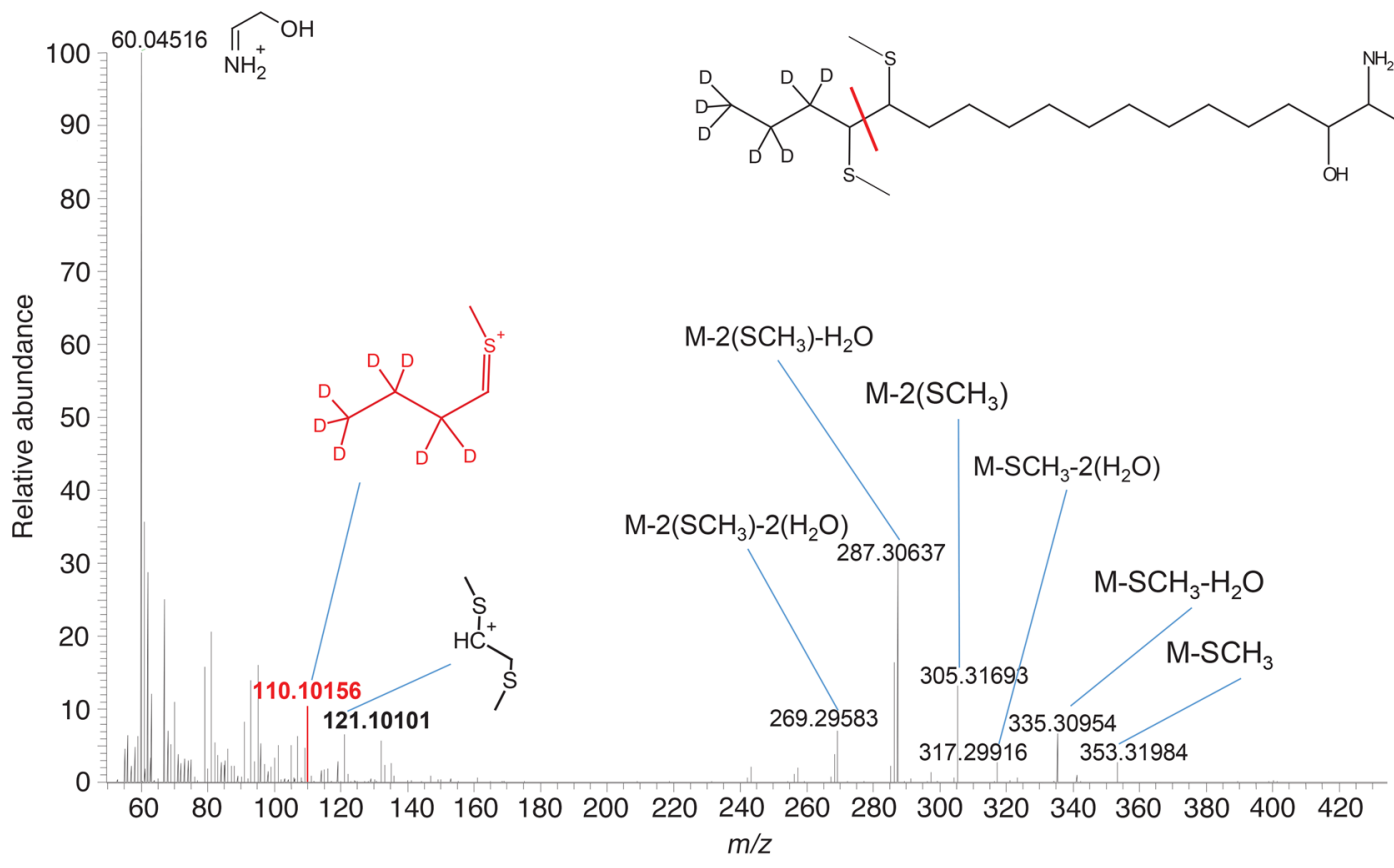

Figure 4. Characterization of a previously unidentified sphingoid base in the DEGS1 disorder. (A) HAP1 WT or DECS1-- cells were cultured in the presence of isotope-labeled d4-serine. Whereas HAP1 WT cells only formed canonical SO+3, DEGS1 ${ }^{-1-}$ cells exclusively formed the SO+3 isomer. Similarly, WT fibroblasts primarily formed canonical $\mathrm{SO}+3$ when cultured in the presence of $\mathrm{d} 4$-serine, while the $\mathrm{SO}+3$ isomer was formed when DECS1 activity was inhibited with 4-HPR. (B) DECS1-- cells were supplemented with isotope-labeled d7SA $(1 \mu \mathrm{M})$ or d7SO $(1 \mu \mathrm{M})$ for 24 hours. The isomeric SO was formed only in d7SA-, but not in d7SO-supplemented cells. (C) Structural analysis of the +7-labeled isomeric $\mathrm{SO}[\mathrm{SO} \Delta$ (?)] after chemical derivatization with dimethyl disulfide. A specific collision fragment with $\mathrm{m} / \mathrm{z} 110.10156$ reflecting the isotope-labeled 4-carbon tail of $\mathrm{SO}+7$ confirmed that the double bond of the isomeric $\mathrm{SO}$ isomer is in the $\Delta 14$ position.

as a potential therapeutic approach for the DEGS1-related disorder. SLs are abundantly present in meat, milk, and egg products (24), whereas plants and yeast usually have phytosphingolipids (phytoSLs) that bear a C4 hydroxyl group instead of the $\Delta 4$ double bond. An increased dietary SL consumption from animal products might therefore increase the levels of unsaturated SL species, thereby lowering the dhSL/SL ratio in the patient. Therefore, we tested whether the SL profile of the patient may be positively influenced by dietary intervention. The pilot study started with a 2-week washout phase providing a primarily plant-based vegetarian diet. Subsequently, the patient was fed with an animal-based diet (milk, eggs, and meat) for another 2 weeks. Plasma samples were taken before and after the washout phase and at the end of the supplementation period. The patient reported no adverse side 
effects except for improved bowel movement during the vegetarian diet. However, the supplementation only had a minor effect on the plasma sphingoid-base profile (Supplemental Figure 2D). We observed a small increase in phytoSL levels after the vegetarian phase and in total SL levels at the end of the supplementation period, but no significant change in the dhSL/SL ratio.

\section{Discussion}

Here, we report on a 22-year-old male patient with a multisystem disease with hypomyelination and degeneration of both the central and the peripheral nervous systems. Leading symptoms were early-onset developmental delay, movement disorder, progressive spasticity, and epilepsy. Similar disease hallmarks may be seen in mitochondriopathies, neuronal ceroid lipofuscinosis, lysosomal storage disorders, or leukodystrophies; however, none of these diagnoses could be confirmed in the index patient. Instead, wholeexome sequencing identified a single nucleotide exchange in DEGS1 (c.893C $>$ T) leading to an amino acid exchange (p.Ala280Val) in a highly conserved region of the respective protein. DEGS1 is a central enzyme in the SL de novo synthesis pathway (Supplemental Figure 3) and has not yet been associated with a human monogenic disease. The enzyme catalyzes the final conversion of dhCer into ceramide by introducing a $\Delta 4,5$ trans double bond into the sphingoid base backbone (25). DEGS1 is a transmembrane protein residing in the ER and contains 3 conserved histidine-based motifs characteristic of membrane lipid desaturases and membrane hydrocarbon hydroxylases $(5,26,27)$. Both pharmacological and genetic ablation of DEGS1 lead to an accumulation of its substrate dhCer (28). Both dhCer and ceramide can be metabolized to complex SLs, although the majority of SLs contains a $\mathrm{SO}(\Delta 4 \mathrm{E})$, whereas SA-based dhSL species are minor. However, lipidomics analysis of the patient plasma revealed significantly elevated dhSL levels, indicating that the desaturase activity in the p.Ala280Val variant is reduced. Elevated levels of dhSL species were also seen in patient-derived fibroblasts and kinetic studies revealed a residual enzyme activity of approximately $20 \%$ for mutant compared with WT protein. Mutant DEGS1 showed a reduced expression in skin-derived fibroblasts and expression levels in transfected HAP1 cells were reproducibly lower than for the WT. Western blot quantification showed an approximately $80 \%$ reduced expression of the mutant protein in patient fibroblasts, which could explain the $20 \%$ residual activity of DEGS1 in these cells.

DEGS2, a homologous isoform of DEGS1 (29), was reported to act as a bifunctional enzyme with either C4-monooxygenase activity adding a hydroxyl group to the $\mathrm{C} 4$ position in forming phytoSLs, or a $\Delta(4)$-desaturase activity similar to DEGS1. However, DEGS2 was not able to compensate for the enzymatic loss in DEGS1-deficient HAP1 cells, indicating mutually exclusive roles for both homologs.

In mice, homozygous deletion of Degs1 (Degs $1^{-/-}$mice) is lethal, although with incomplete penetrance. Surviving pups were small and revealed a complex phenotype including scaly skin and sparse hair, tremor, and metabolic abnormalities (28). Degs1 $1^{-/-}$mice had lower ceramide levels and dramatically more dhCer in blood and tissues compared with WT littermates. Heterozygous Degs $1^{+/-}$ animals showed improved insulin sensitivity on high-fat diet and were resistant to dexamethasone-induced insulin resistance (28). This and subsequent observations indicated that a moderate increase in the dhCer/ceramide ratio is linked to an improved glu- cose metabolism and metabolic control, although the underling physiological mechanisms are not yet fully understood $(30,31)$. Notably, we did not observe a significant difference in the dhSL levels between the heterozygous parents and unrelated controls.

An increased dhSL/SL ratio results in higher rigidity of the plasma membrane (32), which likely affects many biological processes relying on appropriate membrane dynamics for active transport, diffusion, vesicle formation, and signaling. It is tempting to speculate that alterations in the ER structure, as observed in the EM of the sural nerve of the patient, may reflect impaired membrane properties. However, defects in DEGS1 not only influence the dhCer/ceramide ratio but also the profile of complex SLs like (dh)SM and (dh)HexCer, which are formed downstream of dhCer. SM is an abundant component of glia and myelin, and the distinct biophysical properties of dhSM might significantly affect the physiology of glial cells and the structure of the myelin sheath. Indeed, myelin has a special membrane structure with a unique molecular composition and architecture. The most striking features are high levels of plasmalogens and the enrichment of specific glycosphingolipids. Several structural aspects distinguish glycosphingolipids in myelin from glycosphingolipids found in the plasma membrane of most other cell types. In particular, the head groups are primarily based on galactose instead of glucose, a relatively high proportion of hydroxylated fatty acids, and the incorporation of very-long-chain fatty acids with chain lengths up to 26 carbons are characteristic. Even minor perturbations in this molecular composition lead to myelination defects (33), which likely explains the hypomyelination in our patient.

In addition to the increased dhSL levels, we also identified an atypical SL metabolite that seems to be specifically formed under conditions of reduced DEGS1 activity. This metabolite was isomeric to SO but eluted with a slightly shifted retention time compared with canonical SO. This metabolite was prominently present in plasma of the index patient, in DEGS ${ }^{-/-}$HAP1 cells, as well as in fibroblasts treated with the DEGS1 inhibitor 4-HPR. Isotope-labeled d4-serine confirmed that it is a bona fide SL and downstream product of SPT that can be directly formed from d7SA but not from d7SO. Further structural elucidations revealed that this isomeric SO contains a double bond at $\Delta 14$ instead of the $\Delta 4$ position. Sphingoid bases with a $\Delta 14$ double bond have been reported previously. First for sphingadienine, a polyunsaturated downstream metabolite of SO, and recently for 1-deoxySO, an aberrant sphingoid base formed by mutant SPT enzymes in the context of sensory and autonomic neuropathy type 1 (23). In both cases, the $\Delta 14$ double bond was found to be in the $c$ is $(\mathrm{Z})$ conformation, which likewise suggests a $\Delta 14$ double bond of the isomeric SO in cis (Z) conformation [SO(14Z)]. The double bond is likely formed by a not yet identified $\Delta 14-15 Z$ desaturase in an alternative reaction to that catalyzed by DEGS1. The toxicity and pathophysiological relevance of this atypical SO isomer is not clear, but may be a crucial part of the pathomechanism. As a cis (Z) conformation introduces a kink into the structure of the sphingoid base, its lateral assembly with other membrane lipids will differ from canonical SO that contains a straight $(\triangle 4 \mathrm{E})$ double bond. Both the elevation in dhSL species and the SO(14Z) isomer might be relevant for the observed hypomyelination phenotype.

In a pilot experiment we tested whether the serum dhSL/SL ratio could be influenced by a diet rich in canonical SLs. However, 
we were not able to modulate the dhSL/SL ratio in the patient; this 2-week test phase may be too short or the dietary intervention to inefficient to observe a significant metabolic effect.

In summary, we identified DEGS1 as a disease-causing gene implicated in a heritable multisystem disorder with hypomyelination and degeneration of both the central and the peripheral nervous systems. The mutation affects de novo SL synthesis, leading to an altered dhSL/SL ratio and results in the formation of an aberrant and potentially neurotoxic SO isomer.

\section{Methods}

Neuropathology. Resin embedding of the glutaraldehyde-fixed sural nerve biopsy tissue and subsequent electron microscopy was performed using standard procedures (34).

Whole-exome sequencing. Whole-exome sequencing was performed with the DNA from peripheral blood of 5 family members including the index case, 2 unaffected siblings, and both parents. Enrichment was done with an Illumina Enrichment Kit (Nextera Rapid Capture Exome v1.2) and the respective libraries were sequenced on a NextSeq500 sequencer (Illumina). Alignment and variant calling was performed with SeqMule (version 1.2) (35), FastQC (version 0.11.2), BWA-MEM (version 0.7.8-r455), SAMtools (rmdup, version 0.1.19-44428cd), SAMtools (filter, version 0.1.19-44428cd), SAMtools (index, version 0.1.19$44428 \mathrm{~cd}$ ), and GATKLite (realign, version 2.3-9-gdcdccbb). Genome version hg19 was used for the alignment. Three variant callers were applied for variant detection: GATKLite UnifiedGenotyper (variant, version 2.3-9-gdcdccbb); SAMtools (mpileup, version 0.1.19-44428cd); and FreeBayes (version 0.9.14-14-gb00b735). Variants called by at least 2 programs were considered for further analysis. The resulting variant files were combined (GATK, v3.6, CombineVariants) and processed with KGGSeq (v1.0, 14 April, 2017) (36). Variants with a minor allele frequency in public databases (i.e., ExAC, GnomAD, ESP, 1000 Genomes) above $0.75 \%$ were excluded. Average coverage in the target region was $90 \times$ to $142 \times$, with $88 \%-92 \%$ above $20 \times$ coverage. Mutations were confirmed by Sanger sequencing with a BigDye Terminator 3.1 Cycle Sequencing Kit and Genetic Analyzer 3500 (ThermoFisher Scientific).

Cells and cell culture. DEGS1 ${ }^{-/}$and DEGS2-/ HAP1 cells were generated by a commercial service (Horizon Discovery). The introduction of a frame-shift mutation was confirmed by sequencing. HAP1 WT cells were provided as controls. HAP1 cells were cultured in Iscove's Modified Dulbecco's Medium (IMDM, ThermoFisher Scientific) supplemented with $10 \%$ fetal calf serum (FCS), $4 \mathrm{mM} \mathrm{L}$-glutamine, and $1 \%$ penicillin/streptomycin at $37^{\circ} \mathrm{C}$ in $5 \% \mathrm{CO}_{2}$ atmosphere. Patient fibroblasts were derived from a tissue biopsy in the index patient. HeLa (ATCC, CCL-2) and BJ-5ta (ATCC, CRL-4001) cells were cultured in Dulbecco's Modified Eagle's Medium (DMEM, ThermoFisher Scientific) supplemented with $10 \%$ FCS and $1 \%$ penicillin/streptomycin at $37^{\circ} \mathrm{C}$ in $5 \% \mathrm{CO}_{2}$ atmosphere. Transfection of HeLa cells and HAP1 cells was done using Polyjet or GenJet in vitro transfection reagent (SignaGen) according to the manufacturer's protocol.

Immunohistochemistry. Transfected cells were fixed after 24 hours with $4 \%$ paraformaldehyde in PBS. After blocking and permeabilization with $2 \%$ bovine serum albumin, $10 \%$ normal goat serum, and $0.25 \%$ Triton X-100 in PBS for 60 minutes at room temperature, cells were incubated with primary antibodies in blocking solution for 60 minutes at room temperature, washed 3 times in PBS, and incubated with Alexa Fluor 568- or 647-labeled secondary antibodies (1:1,000;
Molecular Probes). DAPI (4',6-diamidino-2-phenylindole; 1:1,000; Invitrogen) was used for nucleic acid staining. Images were taken with a Zeiss Observer Z.1 microscope equipped with an Apotome 2 and HXP 120 lamp. The following primary antibodies were used: mouse anti-PDI (Enzo, ADI-SPA-891, 1:200), mouse anti-Tim23 (BD, 611222, 1:200).

Cycloheximide chase. Transfected HAP1 cells were treated with cycloheximide $(25 \mu \mathrm{g} / \mathrm{ml})$ with or without $10 \mu \mathrm{M}$ MG-132 for the indicated time period. Cells were harvested with cell scrapers in ice-cold PBS and pelleted by centrifugation at $500 \mathrm{~g}$ for 5 minutes.

Western blot. Protein isolation and Western blot were carried out as described elsewhere (37). The primary antibodies used for immunodetection were DEGS1 (Abcam, ab167169; 1:5,000), GFP (Millipore, MAB3580; 1:1,000), and $\alpha$-tubulin (Abcam, ab15246; 1:2,000). As secondary antibodies, horseradish peroxidase-conjugated anti-rabbit (Santa Cruz Biotechnology, sc-2370; 1:10,000) and anti-mouse IgGs (Santa Cruz Biotechnology, sc-2005; 1:10,000) were used. Detection was done using Clarity Western ECL Substrate (Bio-Rad) and a FujiFilm LAS 3000 system. PageRuler or PageRuler Plus Prestained Protein Ladder (ThermoFisher Scientific) was used for protein molecular weight estimation.

Cloning. Human DEGS1 (NM_003676.3) was amplified from a commercially available cDNA library using the following primers: forward, CACCATGGGGAGCCGCGTCTCGCGGGAAGACTTC; reverse, CTCCAGCACCATCTCTCСTTTTTGGTG). The amplicon for DEGS1 lacking the stop codon was subcloned into the pEGFP vector (Clontech). The mutation p.Ala280Val was introduced by targeted mutagenesis. pEF1 $\alpha$-DEGS1-EGFP and DEGS1-EGFP (c.893C >T) were generated by cutting pEGFP-DEGS1 with AflII and EcoRI and blunting with Klenow fragment. pEF1 $\alpha$-Tet3g was digested with EcoRI and HindIII and ends were filled by Klenow fragment followed by dephosphorylation with FastAP. The resulting fragments were ligated with T4 ligase. All inserts were sequence verified using Sanger sequencing.

FACS. Transfected cells were trypsinized, pelleted, and resuspended in PBS containing $250 \mathrm{ng} / \mathrm{ml}$ 7-AAD (BioLegend) for live/dead cell discrimination. Measurements were carried out on a FACSCanto II flow cytometer (Becton Dickinson). For lipidomics, cell pellets were resuspended in PBS containing $250 \mathrm{ng} / \mathrm{ml}$ 7-AAD and 20\% FCS. Sorting was carried out on a FACSAria II cell sorter (Becton Dickinson) and 300,000 GFP-positive and -negative cells were collected per sample.

Lipidomics. Lipid extraction was performed as described previously (38) with some modifications. Plasma sample (20 $\mu \mathrm{l})$ or $0.5-5$ million cells were suspended in $20 \mu \mathrm{l} \mathrm{PBS}$, and $1 \mathrm{ml}$ of a mixture of methanol/MTBE/chloroform (MMC) 4:3:3 (v/v/v) was added. The MMC mix was fortified with $100 \mathrm{pmol} / \mathrm{ml}$ of the following internal standards: d7SA (d18:0), d7SO (d18:1), dhCer (d18:0:12:0), ceramide (d18:1/12:0), glucosylceramide (d18:1/8:0), SM (18:1/12:0), and 50 $\mathrm{pmol} / \mathrm{ml} \mathrm{d7-S1P.} \mathrm{After} \mathrm{brief} \mathrm{vortexing,} \mathrm{the} \mathrm{samples} \mathrm{were} \mathrm{continuously}$ mixed in a Thermomixer (Eppendorf) at $37^{\circ} \mathrm{C}$ (1,400 rpm, 20 minutes). Protein precipitates were obtained after centrifugation (5 minutes, $16,000 \mathrm{~g}, 25^{\circ} \mathrm{C}$ ). The single-phase supernatant was collected, dried under $\mathrm{N}_{2}$, and stored at $-20^{\circ} \mathrm{C}$ until analysis. Before analysis, the dried lipids were dissolved in $100 \mu \mathrm{l}$ methanol.

Liquid chromatography was done as previously described (39) with some modifications. The lipids were separated using a C30 Accucore LC column (ThermoFisher Scientific, $150 \mathrm{~mm} \times 2.1 \mathrm{~mm} \times 2.6 \mu \mathrm{m}$ ) and the following mobile phases: (A) acetonitrile/water (2:8) with 10 $\mathrm{mM}$ ammonium acetate and $0.1 \%$ formic acid, (B) isopropanol/aceto- 
nitrile (9:1) with $10 \mathrm{mM}$ ammonium acetate and $0.1 \%$ formic acid, and (C) methanol at a flow rate of $0.3 \mathrm{ml} / \mathrm{min}$.

The following gradient was applied: 0.0-1.5 minutes (isocratic $70 \%$ A, 20\% B, and 10\% C); 1.5-18.5 minutes (ramp 20\%-100\% B); 18.5-25.5 minutes (isocratic 100\% B); 25.5-30.5 minutes (isocratic $70 \%$ A, $20 \%$ B, and 10\% C).

The liquid chromatography was coupled to a Q-Exactive hybrid quadrupole-orbitrap mass spectrometer (ThermoFisher Scientific); samples were analyzed in positive mode using a heated electrospray ionization (HESI) interface. The following parameters were used: spray voltage $3.5 \mathrm{kV}$, vaporizer temperature $300^{\circ} \mathrm{C}$, sheath gas pressure $20 \mathrm{AU}$, aux gas $8 \mathrm{AU}$, and capillary temperature $320^{\circ} \mathrm{C}$. The detector was set to an $\mathrm{MS}^{2}$ method using data-dependent acquisition with top10 approach with stepped collision energy between 25 and 30. A resolution of 140,000 was used for the full spectrum and a 17,500 for $\mathrm{MS}^{2}$. A dynamic exclusion filter was applied that excludes fragmentation of the same ions for 20 seconds. Identification criteria were (a) resolution with an accuracy of $5 \mathrm{ppm}$ from the predicted mass at a resolving power of 140,000 at 200 , (b) isotopic pattern fitting to expected isotopic distribution, (c) matching retention time on synthetic standards if available, and (d) the specific fragmentation patterns. Quantification was done using single-point calibration. Pooled samples at 4 concentrations were used as quality controls.

Metabolic labeling and sphingoid-base profiling. Cells $(250,000)$ were seeded in $2 \mathrm{ml}$ fresh medium in 6-well plates (BD Falcon) and cultured for 2 days, reaching approximately $70 \%-80 \%$ confluence. The medium was exchanged for L-serine- and L-alanine-free DMEM (Genaxxon Bioscience), containing 10\% FBS (ThermoFisher Scientific; FSA15043) and $1 \%$ penicillin and streptomycin (100 units $/ \mathrm{ml}$ and $0.1 \mathrm{mg} / \mathrm{ml}$, respectively; MilliporeSigma). Two hours after medium exchange, isotope-labeled $\mathrm{d} 3-{ }^{-15} \mathrm{~N}-\mathrm{L}$-serine $(1 \mathrm{mM})$ and $(2,3,3,3)$-d 4 -L-alanine $(2 \mathrm{mM})$ was added (Cambridge Isotope Laboratories). In certain cases, myriocin (Focus Biomolecules) or d7SA/d7SO (Avanti Polar Lipids) was also added to the cells. Palmitate labeling was performed in DMEM (MilliporeSigma) with $10 \% \mathrm{FBS}$ and $1 \%$ penicillin and streptomycin supplemented with $25 \mu \mathrm{M}$ d4-palmitic acid (Cambridge Isotope Laboratories). After 24 hours, cells were harvested in $1 \mathrm{ml}$ cold PBS, counted (Beckman Coulter Z2), pelleted at $600 \mathrm{~g}$ at $4^{\circ} \mathrm{C}$, and stored at $-20^{\circ} \mathrm{C}$ until further processing. During the SPT reaction, 1 deuterium from serine is exchanged with a hydrogen, which results in newly formed sphingoid bases with a $\mathrm{d} 3$ isotope label. In some cases, the quantification of isotope-labeled sphingoid bases was simplified by hydrolyzing the extracted lipids prior to MS analysis (40). During hydrolysis, the conjugated $\mathrm{N}$-acyl chains and attached head groups are removed and the SL backbones released as free sphingoid bases. dhSL species are converted to free SA and unsaturated species to free SO.

Dimethyl disulfide adduct analysis by LC-MS. Dimethyl disulfide (DMDS, $100 \mu \mathrm{l}$ ) and $20 \mu \mathrm{l}$ of $\mathrm{I}_{2}$ (in diethyl ether, $60 \mathrm{mg} / \mathrm{ml}$ ) were add-

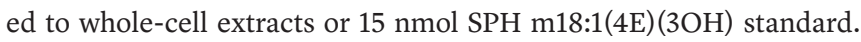
Samples were agitated for 16 hours in an Eppendorf Thermo shaker at $1,400 \mathrm{rpm}$ and $47^{\circ} \mathrm{C}$. The reaction was quenched with $100 \mu \mathrm{l}$ of $5 \%$ aqueous $\mathrm{Na}_{2} \mathrm{~S}_{2} \mathrm{O}_{3}$, extracted with $200 \mu \mathrm{l}$ of hexane, and dried under $\mathrm{N}_{2}$. Samples were dissolved in $50 \mu \mathrm{l}$ methanol/water (1:1) for further analysis according to the method of Dunkelblum, Tan, and Silk (9), with some modifications. The DMDS adducts were separated using a C30 Accucore LC column (ThermoFisher Scientific, $150 \mathrm{~mm} \times 2.1 \mathrm{~mm}$ $\times 2.6 \mu \mathrm{m})$ and the following mobile phases: (A) methanol/water (1:1) with $10 \mathrm{mM}$ ammonium formate and $0.1 \%$ formic acid, and (B) methanol at a flow rate of $0.3 \mathrm{ml} / \mathrm{min}$ using a TLX Transcend pump (ThermoFisher Scientific). The following gradient was applied: (a) 0.0-1.0 minute (isocratic 10\% B), (b) 1.0-26.0 minutes (ramp 10\%-100\% B), (c) 26.0-32.0 minutes (isocratic 100\% B), (d) 32.0-33.0 minutes (ramp 100\%-10\% B), and (e) 33.0-35.0 (isocratic 10\% B).

The liquid chromatography was coupled to a Q-Exactive hybrid quadrupole-orbitrap mass spectrometer; samples were analyzed in positive mode using a HESI interface. The following parameters were used: spray voltage $3.5 \mathrm{kV}$, vaporizer temperature $100^{\circ} \mathrm{C}$, sheath gas pressure $40 \mathrm{AU}$, aux gas $10 \mathrm{AU}$, and capillary temperature $300^{\circ} \mathrm{C}$. The mass spectrometer was operated in 2 alternating scan modes: (a) full scan mode at 140,000 resolution, $3 \times 10^{6}$ AGC target and scan range $150-900 \mathrm{~m} / \mathrm{z}$; and (b) parallel reaction monitoring mode (PRM) at 70,000 resolution, $2 \times 10^{5}$ AGC target, $200 \mathrm{~ms}$ maximum injection time, and $1.5 \mathrm{~m} / z$ isolation window. The normalized collision energy was set to 35, 50, and 70. The inclusion list for the PRM included both the nonlabeled SO DMDS adduct $(\mathrm{m} / \mathrm{z}$ 394.28080) and the d7-labeled SO DMDS adduct $(m / z$ 401.32473).

Statistics. Results are presented as the mean \pm SD or mean $-\mathrm{SD}$ from at least 3 independent experiments using GraphPad Prism 8. Statistical analysis was conducted using a 2-tailed $t$ test or 2-way ANOVA with Tukey's correction for multiple comparisons. A $P$ value of less than 0.05 was considered statistically significant.

Study approval. Written informed consent was obtained from the study participants after approval from the Institutional Review Boards at the participating institutions (Uniklinik RWTH Aachen: EK302-16). Consent was obtained according to the Declaration of Helsinki and consent was given for the publication of patient photographs and video.

\section{Author contributions}

IK, GCK, and TH designed the study. GCK, CK, MM, and ME assessed the phenotype of the patient. M. Bergmann, JMS, and JW performed the neuropathological analysis. Exome sequencing and evaluation was done by M. Begemann, FK, and IK. Lipidomics studies were performed and evaluated by GK, AO, SS, RS, and TH. Cell biological experiments were done by $\mathrm{FK}, \mathrm{NH}$, and $\mathrm{BH}$.

\section{Acknowledgments}

We are grateful to the family participating in the study. We thank Sebastian Gießelmann for excellent technical support. We thank Peter Steuernagel (Klinikum Oldenburg) for initial fibroblast cultures. This work was supported by grants from the German Charcot-Marie-Tooth Disease Network (CMT-Net; 01GM1511D) to JW; funding of the 7th Framework Program of the European Commission (RESOLVE, project number 305707); the Swiss National Foundation (SNF, project 31003A_153390/1); the Hurka Foundation; the Novartis Foundation; and the Rare Disease Initiative Zurich (radiz, Clinical Research Priority Program for Rare Diseases, University of Zurich) to TH.

Address correspondence to: Ingo Kurth, Institute of Human Genetics, Uniklinik RWTH Aachen, Pauwelsstr. 30, 52074 Aachen, Germany. Phone: 49.241.8080178; Email: ikurth@ukaachen.de. Or to: Thorsten Hornemann, Institute for Clinical Chemistry University Hospital Zurich, Rämistrasse 100, 8091 Zürich, Switzerland. Phone: 41.44.255.47.19; Email: thorsten.hornemann@usz.ch. 
1. Hannun YA, Obeid LM. Sphingolipids and their metabolism in physiology and disease. Nat Rev Mol Cell Biol. 2018;19(3):175-191.

2. Hannun YA, Obeid LM. Principles of bioactive lipid signalling: lessons from sphingolipids. Nat Rev Mol Cell Biol. 2008;9(2):139-150.

3. Bikman BT, Summers SA. Ceramides as modulators of cellular and whole-body metabolism. JClin Invest. 2011;121(11):4222-4230.

4. Levy M, Futerman AH. Mammalian ceramide synthases. IUBMB Life. 2010;62(5):347-356.

5. Ternes P, Franke S, Zähringer U, Sperling P, Heinz E. Identification and characterization of a sphingolipid delta 4-desaturase family. J Biol Chem. 2002;277(28):25512-25518.

6. Ogretmen B. Sphingolipid metabolism in cancer signalling and therapy. Nat Rev Cancer. 2018;18(1):33-50.

7. Merrill AH, et al. Sphingolipids--the enigmatic lipid class: biochemistry, physiology, and pathophysiology. Toxicol Appl Pharmacol. 1997;142(1):208-225.

8. Hanada K, et al. Molecular machinery for non-vesicular trafficking of ceramide. Nature. 2003;426(6968):803-809.

9. Bienias K, Fiedorowicz A, Sadowska A, Prokopiuk $\mathrm{S}$, Car H. Regulation of sphingomyelin metabolism. Pharmacol Rep. 2016;68(3):570-581.

10. Adada M, Luberto C, Canals D. Inhibitors of the sphingomyelin cycle: Sphingomyelin synthases and sphingomyelinases. Chem Phys Lipids. 2016;197:45-59.

11. Sabourdy F, et al. Monogenic neurological disorders of sphingolipid metabolism. Biochim Biophys Acta. 2015;1851(8):1040-1051.

12. Astudillo L, et al. Human genetic disorders of sphingolipid biosynthesis. J Inherit Metab Dis. 2015;38(1):65-76.

13. Mosbech MB, et al. Reduced ceramide synthase 2 activity causes progressive myoclonic epilepsy. Ann Clin Transl Neurol. 2014;1(2):88-98.

14. Vanni N, et al. Impairment of ceramide synthesis causes a novel progressive myoclonus epilepsy. Ann Neurol. 2014;76(2):206-212.

15. Ferlazzo E, et al. Autosomal recessive progressive myoclonus epilepsy due to impaired ceramide synthesis. Epileptic Disord. 2016;18(S2):120-127.
16. Fragaki K, et al. Refractory epilepsy and mitochondrial dysfunction due to GM3 synthase deficiency. Eur J Hum Genet. 2013;21(5):528-534.

17. Simpson MA, et al. Infantile-onset symptomatic epilepsy syndrome caused by a homozygous loss-of-function mutation of GM3 synthase. Nat Genet. 2004;36(11):1225-1229.

18. Boukhris A, et al. Alteration of ganglioside biosynthesis responsible for complex hereditary spastic paraplegia. Am J Hum Genet. 2013;93(1):118-123.

19. Janecke AR, et al. Deficiency of the sphingosine1-phosphate lyase SGPL1 is associated with congenital nephrotic syndrome and congenital adrenal calcifications. Hum Mutat. 2017;38(4):365-372.

20. Lovric S, et al. Mutations in sphingosine-1phosphate lyase cause nephrosis with ichthyosis and adrenal insufficiency. J Clin Invest. 2017;127(3):912-928.

21. Prasad R, et al. Sphingosine-1-phosphate lyase mutations cause primary adrenal insufficiency and steroid-resistant nephrotic syndrome. J Clin Invest. 2017;127(3):942-953.

22. Atkinson D, et al. Sphingosine 1-phosphate lyase deficiency causes Charcot-Marie-Tooth neuropathy. Neurology. 2017;88(6):533-542.

23. Steiner R, et al. Elucidating the chemical structure of native 1-deoxysphingosine. J Lipid Res. 2016;57(7):1194-1203.

24. Vesper H, Schmelz EM, Nikolova-Karakashian MN, Dillehay DL, Lynch DV, Merrill AH. Sphingolipids in food and the emerging importance of sphingolipids to nutrition. J Nutr. 1999;129(7):1239-1250.

25. Causeret C, Geeraert L, Van der Hoeven G, Mannaerts GP, Van Veldhoven PP. Further characterization of rat dihydroceramide desaturase: tissue distribution, subcellular localization, and substrate specificity. Lipids. 2000;35(10):1117-1125.

26. Cadena DL, Kurten RC, Gill GN. The product of the MLD gene is a member of the membrane fatty acid desaturase family: overexpression of MLD inhibits EGF receptor biosynthesis. Biochemistry. 1997;36(23):6960-6967.

27. Sperling P, Zähringer U, Heinz E. A sphingolipid desaturase from higher plants. Identification of a new cytochrome b5 fusion protein. $J$ Biol Chem. 1998;273(44):28590-28596.

28. Holland WL, et al. Inhibition of ceramide synthesis ameliorates glucocorticoid-, saturated-fat-, and obesity-induced insulin resistance. Cell Metab. 2007;5(3):167-179.

29. Mizutani Y, Kihara A, Igarashi Y. Identification of the human sphingolipid C4-hydroxylase, hDES2, and its up-regulation during keratinocyte differentiation. FEBS Lett. 2004;563(1-3):93-97.

30. Holland WL, Summers SA. Strong heart, low ceramides. Diabetes. 2018;67(8):1457-1460.

31. Summers SA. Could ceramides become the new cholesterol? Cell Metab. 2018;27(2):276-280.

32. Vieira CR, et al. Dihydrosphingomyelin impairs HIV-1 infection by rigidifying liquid-ordered membrane domains. Chem Biol. 2010;17(7):766-775.

33. Aggarwal S, Yurlova L, Simons M. Central nervous system myelin: structure, synthesis and assembly. Trends Cell Biol. 2011;21(10):585-593.

34. Nolte KW, Hans VJ, Schattenfroh C, Weis J, Schröder JM. Perineurial cells filled with collagen in 'atypical' Cogan's syndrome. Acta Neuropathol. 2008;115(5):589-596

35. Guo Y, Ding X, Shen Y, Lyon GJ, Wang K. SeqMule: automated pipeline for analysis of human exome/genome sequencing data. Sci Rep. 2015;5:14283.

36. Li MX, et al. Predicting mendelian disease-causing non-synonymous single nucleotide variants in exome sequencing studies. PLoS Genet. 2013;9(1):e1003143.

37. Esmaeili M, et al. The tumor suppressor ING1b is a novel corepressor for the androgen receptor and induces cellular senescence in prostate cancer cells. J Mol Cell Biol. 2016;8(3):207-220.

38. Pellegrino RM, Di Veroli A, Valeri A, Goracci L, Cruciani G. LC/MS lipid profiling from human serum: a new method for global lipid extraction. Anal Bioanal Chem. 2014;406(30):7937-7948.

39. Narváez-Rivas $M$, Zhang Q. Comprehensive untargeted lipidomic analysis using core-shell C30 particle column and high field orbitrap mass spectrometer.JChromatogr A. 2016;1440:123-134

40. Alecu I, et al. Cytotoxic 1-deoxysphingolipids are metabolized by a cytochrome P450-dependent pathway. JLipid Res. 2017;58(1):60-71. 